\title{
Markers of activated inflammatory cells correlate with severity of liver damage in children with nonalcoholic fatty liver disease
}

\author{
RITA DE VITO $^{1^{*}}$, ANNA ALISI ${ }^{2 *}$, ANDREA MASOTTI $^{3}$, SARA CECCARELLI $^{2}$, NADIA PANERA $^{2}$, \\ ARIANNA CITTI ${ }^{1}$, MICHELE SALATA ${ }^{4}$, LUCA VALENTI ${ }^{5}$, ARIEL E. FELDSTEIN ${ }^{6}$ and VALERIO NOBILI ${ }^{2,6}$ \\ ${ }^{1}$ Unit of Pathology, ${ }^{2}$ Unit of Liver Research, ${ }^{3}$ Gene Expression-Microarrays Laboratory, ${ }^{4}$ Department of Pediatrics of \\ 'Bambino Gesù' Children's Hospital, IRCCS, Rome; ${ }^{5}$ Department of Internal Medicine, Università degli Studi, \\ Ospedale Maggiore Policlinico 'Ca' Granda' IRCCS, Milan, Italy; ${ }^{6}$ Department of Pediatrics, \\ University of California, San Diego, CA, USA
}

Received January 5, 2012; Accepted February 13, 2012

DOI: 10.3892/ijmm.2012.965

\begin{abstract}
Concomitantly to the obesity epidemic, nonalcoholic fatty liver disease (NAFLD) has become the leading cause of liver disease in children. NAFLD encompasses a spectrum of histological damage ranging from simple steatosis to nonalcoholic steatohepatitis (NASH), with possible progression to cirrhosis. There is growing evidence that the immune system plays a pivotal role in the initiation and progression to NASH but the cellular nature of the hepatic inflammation is still unknown. The present study includes 34 children with biopsy-proven NAFLD. Liver damage was evaluated by the NAFLD activity score (NAS), and the inflammatory infiltrate was characterized by immunohistochemistry for CD45, CD3 and CD163 which are markers of leukocytes, $\mathrm{T}$ cells and activated Kupffer cells/macrophages, respectively. Our results have shown that $\mathrm{CD}^{+} 5^{+}(\mathrm{P}<0.0001)$ and $\mathrm{CD}^{2} 63^{+}(\mathrm{P}<0.0001)$ cells were markedly increased in children with severe histological activity (NAS $\geq 5$ ) compared to children with lower activity (NAS $<5$ ), whereas $\mathrm{CD}^{+}$cells were significantly lower $(\mathrm{P}<0.01)$ in children with severe histological activity. There was a significant association between the numbers of $\mathrm{CD} 45^{+}, \mathrm{CD}^{+}$and $\mathrm{CD} 163^{+}$cells, regarding both the portal tract and liver lobule, and the severity of steatosis, ballooning and fibrosis $(\mathrm{P}<0.01)$. These data suggest that the severity
\end{abstract}

Correspondence to: Dr Valerio Nobili, Hepato-Metabolic Disease Unit and Unit of Liver Research at 'Bambino Gesù' Children's Hospital, IRCCS, P.le S. Onofrio 4, I-00165 Rome, Italy

E-mail: nobili66@yahoo.it

Dr Anna Alisi, Research Laboratories, Unit of Liver Research at 'Bambino Gesù' Children's Hospital, IRCCS, P.le S. Onofrio 4, I-00165 Rome, Italy

E-mail: anna.alisi@opbg.net

${ }^{*}$ Contributed equally

Key words: nonalcoholic fatty liver disease, nonalcoholic steatohepatitis, Kupffer cells, CD3, CD45, CD163, children and composition of the inflammatory infiltrate correlate with steatosis and the severity of disease in children with NAFLD. Moreover, a decrease in $\mathrm{CD}^{+}$cells may be involved in the pathogenesis of liver damage. Future studies should evaluate whether it can predict the progression of liver disease independently of established histological scores.

\section{Introduction}

Nonalcoholic fatty liver disease (NAFLD) is an increasingly recognized cause of liver-related morbidity and mortality in children $(1,2)$. At present, the prevalence of NAFLD is estimated at a value of $3-10 \%$ in children of westernized countries. Nevertheless, due to its correlation with the epidemic pediatric obesity, this percentage increases up to $50-70 \%$ in obese subjects $(3,4)$. As in adults, pediatric NAFLD includes a spectrum of diseases ranging from fatty liver to nonalcoholic steatohepatitis (NASH), which may progress to fibrosis and its complications (5-7).

Early diagnosis to evaluate grade and stage of the disease, as well as effective and safe therapeutic approaches for the more advanced state of NASH (i.e. the presence of mild and severe fibrosis) are urgently required to counterbalance the escalation of pediatric NAFLD $(8,9)$. Most therapeutic approches, already available or under investigation, target the major pathways which have been implicated in the pathogenesis of NAFLD/NASH (10-12). Intrahepatic fat accumulation and insulin resistance represent the primary events promoting fatty liver, which in turn may progress to NASH by a complicated network of secondary hits, including pro-inflammatory molecules, oxidative stress response, increased cell death by apoptosis and other signals capable to sustain the axis gutadipose tissue-liver $(10,12,13)$.

Furthermore, growing evidence demonstrates that NAFLD, similarly to other liver diseases, is characterized by intricate interactions between resident and recruited cells which may determine the type and severity of liver damage. Particularly there is an increasing recognition of the potential role of macrophages in danger recognition, immune tolerance response and derangement of lipid homeostasis that characterize NAFLD (14). Rivera et al (17) have reported that Kupffer 
cell depletion, in mice on a methionine and choline deficient (MCD) diet (an established experimental model of NASH), prevented hepatic fat accumulation and liver damage. Kupffer cells are also important regulators of the biological exchanges between hepatocytes and other liver cells; they engage and sustain the action of neutrophils, natural killer T lymphocytes (NKT) and blood monocyte-derived macrophages; they phagocytose and remove microorganisms, apoptotic cells and cell debris; besides they process and present antigens to attract cytotoxic and regulatory $\mathrm{T}$ cells contributing to the adaptive immunity (18). Several studies demonstrated that failure of Kupffer cells to properly exert these functions play a critical role in various forms of liver injury (19-21). Thus Kupffer cells may contribute both to the development of fatty liver and its progression to NASH $(22,23)$. In fact an increased gut-derived endotoxemia may activate Kupffer cells via Tolllike receptor 4 (TLR-4) signaling. The interaction between TLR-4 and endotoxin results in the release of a myriad of pro-inflammatory mediators which induce hepatic injury and fibrosis (24). Recently, Kremer et al (25) demonstrated that hepatosteatosis, in mice fed an MCD diet, is characterized at the hepatic level by an induction of interferon- $\gamma$ and tumor necrosis factor- $\alpha$, an elevated content of interleukin (IL)-12 and a remarkable reduction in the number and activity of NKT cells. The authors also highlighted that the reduction of the NKT cell population is due to the activation of Kupffer cells via an endotoxin-mediated mechanism. Furthermore, Stienstra et al (26) demonstrated a relevant role of the Kupffer cells in hepatic triglyceride storage, promoting hepatic steatosis by means of IL-1 $\beta$-mediated suppression of peroxisome proliferator-activated receptor $\alpha$ (PPAR- $\alpha$ ) activity.

Based on these findings increased levels of activated Kupffer cells and consequently reduced levels of NKT may be a novel interesting molecular pattern to define the severity of biological alterations underpinning the progression of liver damage in NAFLD.

Here we used three different surface markers (CD45, CD3 and CD163) to investigate whether the cellular nature of inflammatory infiltrate in liver tissue, from children with the whole spectrum of NAFLD, is associated with the severity of liver damage. CD45, known as common leukocyte antigen, is one of the most abundant leukocyte cell surface glycoproteins and its expression is restricted to haematopoietic cells (27). CD3 antigen represents the most specific as well the most sensitive T cells lineage marker, including NK and NKT (27). CD163 is a member of the cysteine scavenger receptor superfamily that is expressed in the cells of monocyte/macrophage origin, including Kupffer cells (28). It is also expressed in resident scavenger cells such as those derived from monocyte/macrophage (29). Our results suggest a correlation between numbers of $\mathrm{CD}^{+}, \mathrm{CD} 63^{+}$and $\mathrm{CD} 45^{+}$cells and the severity of NAFLD.

\section{Materials and methods}

Patients and laboratory/clinical data. In this study we analyzed 34 archival liver samples derived from our cohort of well-characterized subjects with liver biopsy-proven NAFLD. Indications for liver biopsy in this series were previously reported (1). The samples were derived from patients referred from January 2007 to March 2008 to the Hepato-Metabolic Disease Unit of Bambino Gesù Children's Hospital, Rome, Italy. Exclusion criteria included: alcohol and drugs (e.g. valproate, amiodarone or prednisone) intake, the presence of hepatic viral infections (Hepatitis A, B, C, D, E and G, cytomegalovirus and EpsteinBarr virus) and a history of parenteral nutrition at the time of biopsy. Autoimmune liver disease, metabolic liver disease, Wilson's disease, and $\alpha$-1-antitrypsin-associated liver disease were ruled out using standard clinical, laboratory and histological criteria. The study was approved by the Ethics Committee of the 'Bambino Gesù' Children's Hospital and informed consent was obtained from each patient or responsible guardian.

Weight and height were measured using standard procedures. Body mass index (BMI) was calculated and converted to standard deviation scores (SDS) using US reference data (30). Waist circumference was measured at the highest point of the iliac crest.

Alanine (ALT), aspartate (AST), and $\gamma$-glutamyl-(GGT) aminotransferase, total triglycerides and total cholesterol were evaluated using standard laboratory methods. Insulin was measured by radioimmunoassay (Myria Technogenetics, Milan, Italy). Glucose and insulin were measured at 0, 30, 60, 90 and 120 min during an oral glucose tolerance test performed with $1.75 \mathrm{~g}$ glucose $/ \mathrm{kg}$ of body weight (up to $75 \mathrm{~g}$ ). The severity of insulin resistance and sensitivity were determined, respectively, by the homeostatic model assessment insulin resistance (HOMA-IR) using the formula: insulin resistance $=$ (insulin $\mathrm{x}$ glucose)/22.5 and by the insulin sensitivity index (ISI) derived from oral glucose tolerance test (OGGT) using the formula: ISI $=(10,000 /$ square root of [fasting glucose $\mathrm{x}$ fasting insulin] $\mathrm{x}$ [mean glucose $\mathrm{x}$ mean insulin during OGTT]) $(31,32)$.

Liver histopathology. Tissue samples obtained from liver biopsy were fixed in buffered formalin embedded in paraffin and sliced into $3 \mu \mathrm{m}$ sections. Standard histological stains included haematoxilyn and eosin (H\&E), Periodic Schiff Acid without and with diastase (PAS/PAS-D) and Van Gieson trichrome stain. Steatosis, necro-inflammation, hepatocyte ballooning and fibrosis were scored according to the NAFLD Clinical Research Network criteria. Features of steatosis (0-3), lobular inflammation (0-3) and hepatocyte ballooning (0-2) were combined to obtain the NAFLD activity score (NAS) (33). In this study we subdivided cases in two subgroups according to the severity of liver disease assessed as NAS score (NAS $\geq 5$ or $<5$ ). Portal chronic inflammation was also evaluated (0-1). Fibrosis was scored as 0 , none; 1 , periportal or perisinusoidal fibrosis; 2 , perisinusoidal and portal/periportal fibrosis; 3, bridging fibrosis; and 4, cirrhosis (33).

Immunohistochemistry. Immunostaining of formalin-fixed paraffin-embedded tissue specimens was performed after dewaxing and rehydrating $3 \mu \mathrm{m}$ thick sections. Endogenous peroxidase was blocked with $10 \%$ hydrogen peroxide followed by incubation on avidin/biotin blocking system (Thermo Scientific) to inhibit endogenous biotin activity. We used primary monoclonal mouse antibodies raised against: CD45 (clone PD7/26 and 2B11; Dako, Carpinteria, CA), CD3 (clone PS1; Novocastra, New Castle, UK) and CD163 (clone 10D6; Novocastra). Heat-induced epitope retrieval was performed by boiling the slides with Dako Target Retrieval Solution (10X). Staining was performed with Dako Envision Plus System (Dako Cytomation). 


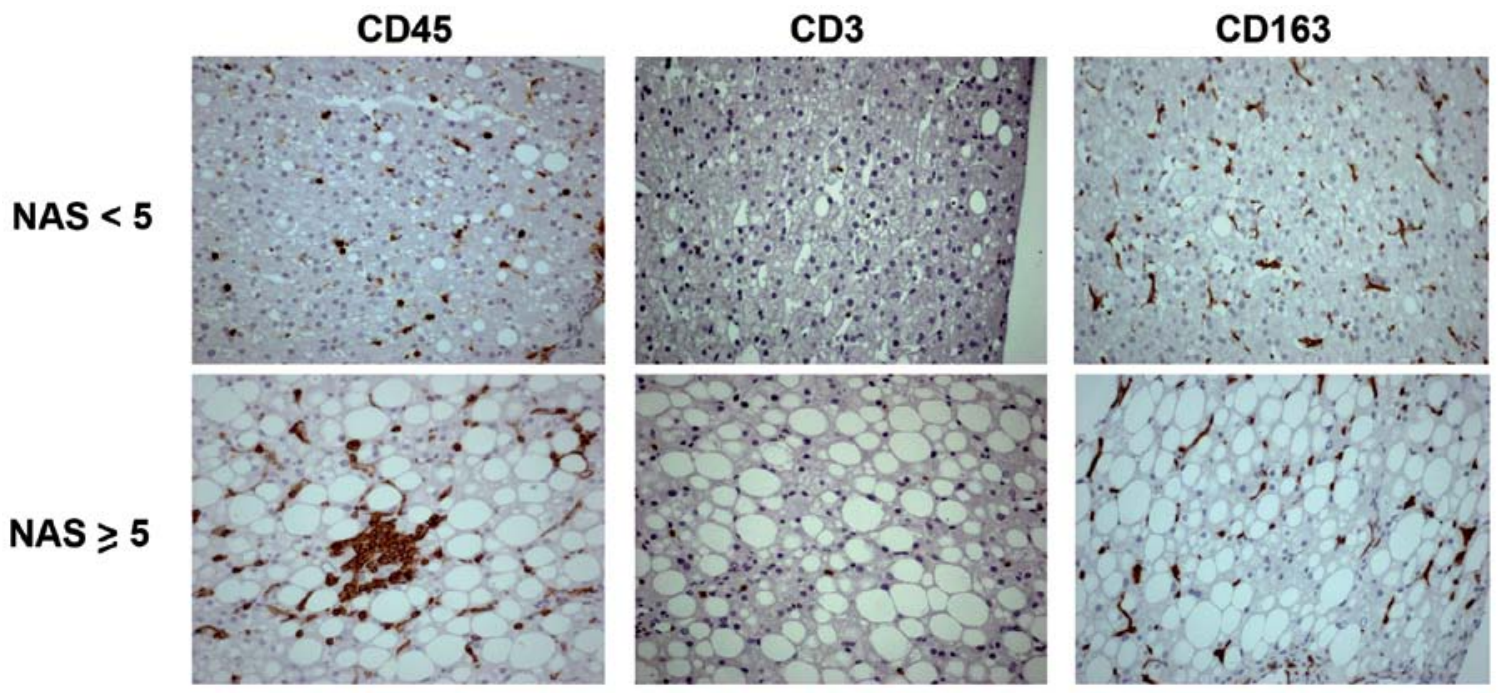

Figure 1. Differential profile of inflammatory cell infiltration in the liver of children with NAFLD. Immunohistochemical evaluation of $\mathrm{CD}^{+} 5^{+}$, $\mathrm{CD}^{+}{ }^{+}$and $\mathrm{CD}_{163}{ }^{+}$cells in the liver tissues of children with NAFLD according to NAS $<5$, and NAS $\geq 5$. Magnification, $\mathrm{x} 200$.

The density of cells within the portal tract and liver lobule which stained positive to the various monoclonal antibodies was determined by counting the number of positive cells in ten random portal tracts and in ten areas of lobular region at a magnification of x200 under light microscopy (Zeiss Imager A1).

Statistical analysis. Normality distribution was preliminary assessed by the Shapiro-Wilk test. Continuous variables are given as median, interquartile range (IQR) and minimum and maximum values because of skewed distributions. Since data were not normally distributed, between-group comparisons of continuous variables were performed by the exact Mann-Whitney test. Spearman's correlation coefficients were estimated: between the number of portal, or lobular $\mathrm{CD}_{4} 5^{+}, \mathrm{CD}^{+}$and $\mathrm{CD} 163^{+}$cells and portal or lobular inflammation; and between the number of portal plus lobular $\mathrm{CD} 45, \mathrm{CD}^{+}$and $\mathrm{CD}_{163}{ }^{+}$cells and NAFLD features, including steatosis, ballooning, fibrosis and NAS.

Statistical significance was set to a P-value $<0.05$. Statistical analyses were performed by SPSS statistical software (SPSS Inc., Chicago, IL, USA).

\section{Results}

Patient characteristics and histological features. Clinical features of the patients included in this study are shown in Table I. Out of 34 patients, 23 were males and 11 females with a median age of 10.21 years (range 5.83-17.59 years). Mild-moderate steatosis was evident in 25 subjects $(73.5 \%)$ while the remaining 9 (26.5\%) presented severe steatosis. Thirty-two (94.1\%) and 16 children (47.1\%), respectively showed mild-moderate inflammation and ballooning. Mildmoderate perisinusoidal or periportal fibrosis was observed in 13 children (38.2\%), 5 (14.7\%) had perisinusoidal and portal/ periportal fibrosis, and 4 (11.8\%) bridging fibrosis.

Differential profile of inflammatory cell infiltration in the liver of children with the spectrum of NAFLD. Based on the NAS score, patients were divided into two distinct groups according to the severity of NAS score (NAS $<5$ or $\geq 5$ ). The
Table I. Liver histopathology of the 34 study children with NAFLD.

Score

\begin{tabular}{lllll}
\hline 0 & 1 & 2 & 3
\end{tabular}

\begin{tabular}{lcrrrr}
\hline \multicolumn{2}{l}{ Histological features } & & & & \\
Steatosis & - & $13(38.2)$ & $12(35.3)$ & $9(26.5)$ & - \\
Inflammation & $2(5.9)$ & $21(61.8)$ & $11(32.3)$ & - & - \\
Ballooning & $18(52.9)$ & $9(26.5)$ & $7(20.6)$ & - & - \\
Fibrosis & $12(35.3)$ & $13(38.2)$ & $5(14.7)$ & $4(11.8)$ & 0
\end{tabular}

Results are presented as the number and percentage $(\%)$ of patients.

main clinical and laboratory data of patients are described in Table II. Children with NAS $\geq 5$ had significantly higher waist circumference, ALT and GGT levels compared to those without NASH $(\mathrm{P}<0.05)$. Furthermore, patients with NAS $\geq 5$ had significantly higher triglyceride levels and fibrosis scores compared to those with NAS $<5(\mathrm{P}<0.05)$. The different groups were similar with regards to their BMI, total cholesterol and triglycerides, OGTT, HOMA-IR and ISI.

To quantify the inflammatory cell infiltration the number of the $\mathrm{CD}_{4} 5^{+}, \mathrm{CD}^{+}$and $\mathrm{CD}_{163}{ }^{+}$cells were counted in all available fields, then the mean value was calculated. As shown in Table II, we observed a significant increase in the number of $\mathrm{CD}^{+} 5^{+}(\mathrm{P}<0.0001)$ and $\mathrm{CD} 163^{+}(\mathrm{P}<0.0001)$ cells in children with NAS $\geq 5$. On the other hand, the number of $\mathrm{CD}^{+}$cells was significantly lower $(\mathrm{P}<0.01)$ in children with $\mathrm{NAS} \geq 5$ than in children with NAS $<5$. These results were also strongly evident by the qualitative evaluation of the staining with antiCD45, CD3 and CD163 antibodies (Fig. 1).

Hepatic CD45, CD3, and CD163 expression correlates with disease severity in children with NAFLD. We next tested 


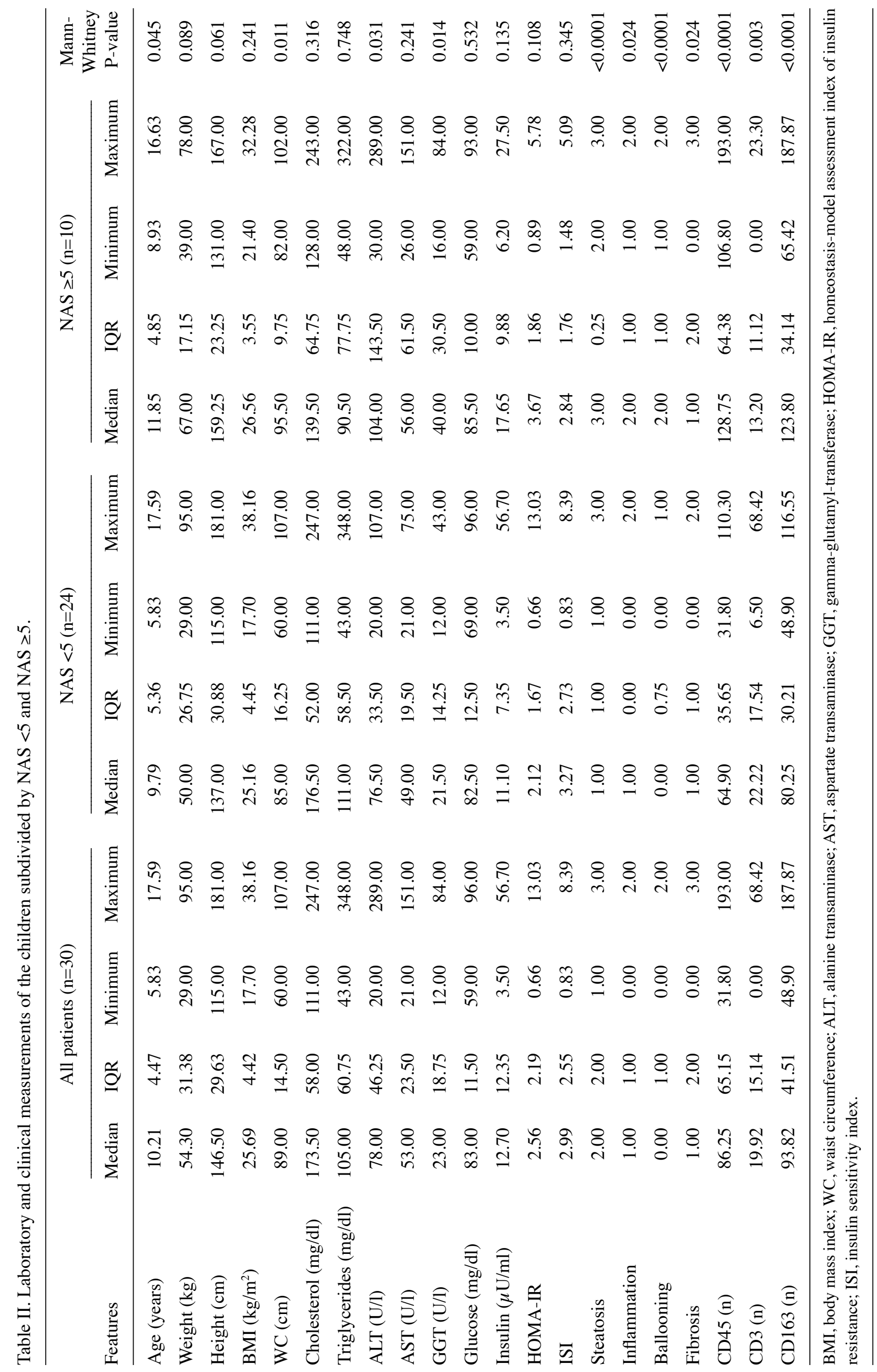


Table III. Correlation between the number of $\mathrm{CD}_{4} 5^{+}, \mathrm{CD}^{+}$and $\mathrm{CD} 163^{+}$cells in portal or lobular regions, and portal or lobular inflammation, respectively.

\begin{tabular}{|c|c|c|c|c|c|c|}
\hline & \multicolumn{2}{|l|}{ CD45 } & \multicolumn{2}{|l|}{ CD3 } & \multicolumn{2}{|l|}{ CD163 } \\
\hline & Correlation coefficient & P-value & Correlation coefficient & P-value & Correlation coefficient & P-value \\
\hline Portal inflammation & 0.336 & 0.049 & -0.391 & 0.022 & 0.472 & 0.005 \\
\hline Lobular inflammation & 0.555 & 0.001 & -0.618 & $<0.001$ & 0.489 & 0.003 \\
\hline
\end{tabular}

Table IV. Correlation between the number of $\mathrm{CD}_{4} 5^{+}, \mathrm{CD}^{+}$and $\mathrm{CD} 163^{+}$cells in portal and lobular regions (considered as sum) and steatosis, ballooning and fibrosis.

\begin{tabular}{|c|c|c|c|c|c|c|}
\hline & \multicolumn{2}{|l|}{ CD45 } & \multicolumn{2}{|l|}{ CD3 } & \multicolumn{2}{|l|}{ CD163 } \\
\hline & Correlation coefficient & P-value & Correlation coefficient & P-value & Correlation coefficient & P-value \\
\hline Steatosis & 0.842 & $<0.001$ & -0.702 & $<0.001$ & 0.755 & $<0.001$ \\
\hline Ballooning & 0.623 & $<0.001$ & -0.459 & 0.006 & 0.496 & 0.003 \\
\hline Fibrosis & 0.442 & 0.009 & -0.350 & 0.043 & 0.356 & 0.039 \\
\hline
\end{tabular}
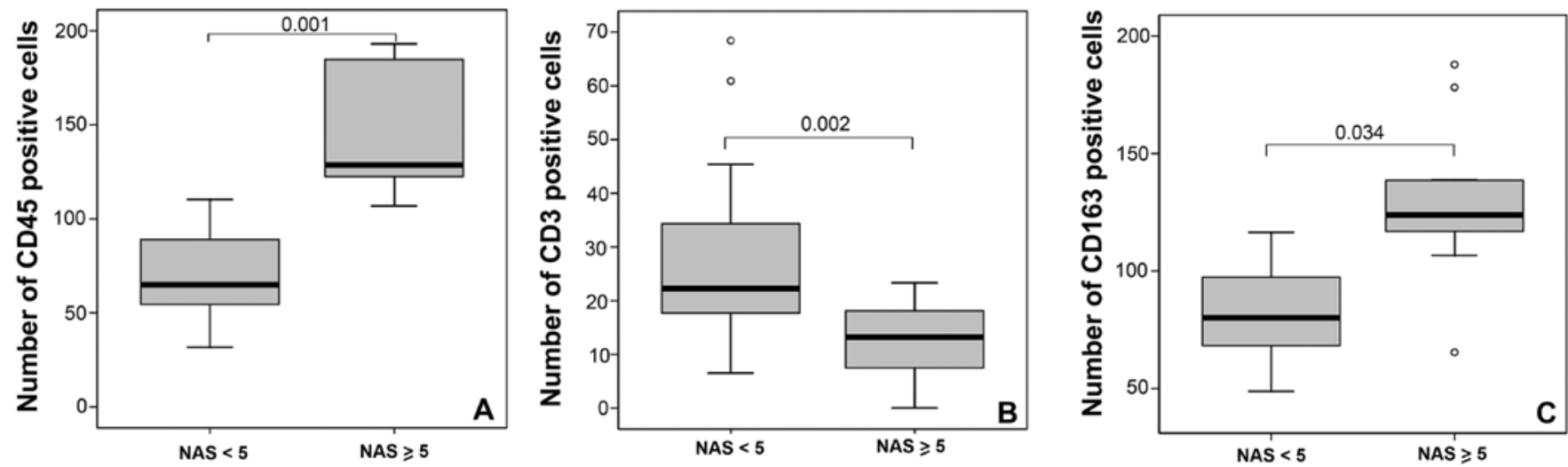

Figure 2. Distribution of CD45, CD3 and CD163 intrahepatic positivity with respect to NAS. Significant differences in number of (A) $\mathrm{CD}_{4} 5^{+},(\mathrm{B}) \mathrm{CD}^{+}$and (C) $\mathrm{CD}_{163^{+}}$cells in 34 children with NAFLD according to NAS $<5$, and NAS $\geq 5$. Statistical significance, calculated as Student's t-test, was reported.

whether the number of $\mathrm{CD} 45^{+}, \mathrm{CD}^{+}$or $\mathrm{CD} 163^{+}$cells was associated with the severity of the histopathological features in children with NAFLD, including degree of steatosis, inflammation, ballooning and stage of fibrosis. Based on our previous study (34), suggesting the relevance of the portal chronic inflammation in defining disease severity in Italian children with NAFLD, we correlated the $\mathrm{CD} 45^{+}, \mathrm{CD}^{+}$and $\mathrm{CD} 163^{+}$cell number in portal and lobular regions with portal and lobular inflammation, respectively. As shown in Table III, the number of $\mathrm{CD}_{4} 5^{+}, \mathrm{CD}^{+}$and $\mathrm{C}_{163}{ }^{+}$cells, either in portal or in lobular spaces, was significantly correlated with both portal and lobular inflammation, with CD3 showing a very strong negative correlation with these indices. These results suggest that the number of portal and lobular inflammatory cells is associated with liver damage in NAFLD and the sum of these two values may correlate with other histological aspect of disease. Noteworthy, we found a statistically significant association of all markers with steatosis, ballooning and fibrosis $(\mathrm{P}<0.01)$ (Table IV).
Significant differences in the number of $\mathrm{CD} 45^{+}, \mathrm{CD}^{+}$and $\mathrm{CD}_{163}{ }^{+}$cells between children with NAS $<5$ and NAS $\geq 5$ were observed (Fig. 2). In addition, the composition of the inflammatory infiltrate (higher $\mathrm{CD}_{4} 5^{+}$and $\mathrm{CD}_{163}{ }^{+}$cells, and lower $\mathrm{CD}^{+}$ cells) was also associated with the presence of fibrosis (Table V). However, at an exploratory logistic regression analysis, after correction for the NAS score, a higher number of intralobular $\mathrm{CD}^{+}$cells was nominally associated with the presence of liver fibrosis (OR, 1.56; 95\% CI, 1.02-2.84; $\mathrm{P}=0.04)$. Due to the strong monotonous association with NAS and the limited number of subjects considered, the independent effect of other cellular infiltrates on NAS could not be evaluated in this pilot study.

\section{Discussion}

A growing number of evidence supports a role for the immune system and inflammatory cells as potential players in the pathogenesis of fatty liver and its progression to NASH 
Table V. Association between histological parameters of disease activity and the presence of fibrosis in 34 children with NAFLD.

\begin{tabular}{|c|c|c|c|}
\hline & \multicolumn{2}{|c|}{ Fibrosis } & \multirow[b]{2}{*}{ P-value } \\
\hline & $\begin{array}{l}\text { Absent } \\
(n=12)\end{array}$ & $\begin{array}{l}\text { Present } \\
(n=22)\end{array}$ & \\
\hline Steatosis (1-3) & $1(1-2)$ & $2(2-3)$ & 0.012 \\
\hline Necroinflammation (0-3) & $1(1-1)$ & $1(1-2)$ & 0.010 \\
\hline Ballooning (0-2) & $0(0-1)$ & $1(0-2)$ & 0.096 \\
\hline NAS (1-8) & $3(2-3)$ & $4(3-6)$ & 0.004 \\
\hline $\mathrm{CD}_{4} 5^{+}$cells ${ }^{\mathrm{a}}$ & $65.0(54-84)$ & $96.4(62-126)$ & 0.035 \\
\hline $\mathrm{CD}_{163^{+}}$cells $^{\mathrm{a}}$ & $78.8(62-94)$ & $106(80-124)$ & 0.019 \\
\hline $\mathrm{CD}^{+}$cells $^{\mathrm{a}}$ & $24.6(18-40)$ & $18.1(2-23)$ & 0.047 \\
\hline
\end{tabular}

Median and (interquartile range) values are shown; ${ }^{\text {acounted in ten portal }}$ tracts and in ten areas of lobular region at a magnification of x200 under light microscopy; ${ }^{\mathrm{b}} \mathrm{P}$-value of fibrosis for the Wilcoxon test.

$(14,21,35)$. Increasing levels of both tumor necrosis factor- $\alpha$ and interferon- $\gamma$, and activation of cells of the innate immune system, have been reported in various NAFLD models and patients with the disease (36-39). This phenomenon seems to be relevant both in the systemic and in the intrahepatic circulation (40).

Resident inflammatory cells exert several functions during NAFLD development. In fact, the inflammation and the increase of inflammatory cells, such as Kupffer and NKT cells, may represent a defensive response to the hepatic injury caused by fatty liver but also an early signal for the development of fibrosis $(41,42)$. These findings suggest that the characterization of the quantity and type of the inflammatory infiltrate could be a novel molecular marker to define tissue damage in NAFLD as well as to identify potential predictors of fibrosis.

In this study we characterized the inflammatory infiltrate in liver tissue from children with NAFLD by the analysis of cells expressing CD45, CD3 and CD163 markers. We found that the number of CD45, CD3 and CD163 liver-resident cells was altered in patients with more severe histological activity and correlated with the severity of chronic liver damage, as indicated by the presence of fibrosis. CD45 is uniformly distributed in the plasma membrane of all hematopoietic cells, suggesting that its increase may represent the first indicator that one or more type of inflammatory cells rise with severity of NASH and fibrosis. Therefore, as necroinflammation is histologically defined based on the number of inflammatory lobular cells (33), an increase in CD $45^{+}$cells could be expected. Interestingly, we found that the number of liver resident CD163 ${ }^{+}$ Kupffer cells increased in children with NASH and correlated with severity of disease. These results fit well with previous studies suggesting that Kupffer cell activation is a causal factor for hepatic damage in NASH (14-16). Furthermore, our recent data indicated that the hepatic expression of $\mathrm{CD}_{163}{ }^{+}$cells is increased in a rodent model of diet-induced NAFLD (43).

The increased expression of both CD163 and soluble CD163 (sCD163) in activated Kupffer cells has been reported in several studies regarding human liver diseases (44-46). Nevertheless, in a recent article Bauer et al (47) report no differences between CD163 or sCD163 in fatty liver when compared to healthy controls. This similarity of CD163 levels in steatotic and in non-steatotic human liver could be explained by the limited number of liver samples (47). Furthermore, it is known that CD163 is shed in its soluble form by inflammatory stimuli making sCD163 a specific marker of macrophage activation (48). In view of literature evidence and of the data here reported, it would be interesting to analyze the SCD163 levels in NAFLD patients in order to better delineate new possible parameters for classifying the severity and progression of liver damage.

Recently, Tajiri et al (40), demonstrated that $\mathrm{CD}^{+} / \mathrm{CD}^{2} 6^{+}$ NKT cells are increased in NAFLD patients as NAS is augmented. On the contrary, although an association between intrahepatic $\mathrm{CD}^{+}$cells and NAS was detected and a pilot analysis also showed an independent association between intralobular $\mathrm{CD}^{+}$cells and fibrosis, we also found a statistically relevant decrease of $\mathrm{CD}^{+}$inflammatory cell number in NASH children. These data are in line with experimental data indicating that steatosis may be associated with a reduction of hepatic NKT cells, determining an alteration in cytokine homeostasis with an increased release of pro-inflammatory cytokines which render the liver more susceptible to noxious insults (21). An inverse correlation was reported between the severity of steatosis and NKT cell number in genetic and acquired NAFLD animal models $(49,50)$, and peripheral NKT cells have been reported to be decreased in humans with NAFLD (51). The reduced NKT cell number and altered activation status, correlated with an increased hepatic production of Th1 cytokines (such as Tumor necrosis factor- $\alpha$, IL-12 and interferon- $\gamma$ ) and was involved in the pathogenesis of liver damage in NASH, possibly due to decreased secretion of the Th2 cytokine IL-4 (52). The mechanisms underpinning the decrease in NKT cells in NAFLD are still unknown. Since IL-12 has been shown to increase NKT cell activationinduced cell death (52), abnormal Kupffer cell activation due to increased LPS, with consequent increase in IL-12, has been proposed to play a role (25). Additional studies should be performed to evaluate whether the reduction in $\mathrm{CD}^{+}$cells is specific for NKT or more generalized, and whether, on the contrary, a selective increase in intralobular $\mathrm{CD}^{+}$cells is associated with the progression of liver disease.

The current study has some limitations, including a relatively small sample size and the lack of a matched control group of children without liver disease. Nevertheless, the study also has strengths, as it represents the first report on the intrahepatic infiltrating immune cells in children with NAFLD. We assessed a variety of different markers of innate immune cells in a group of children with biopsy-proven NAFLD representing the entire spectrum of disease severity, in whom extensive characterization of a metabolic phenotype was performed. The extension of the study to a larger cohort of patients, as well as the evaluation of the possible involvement of other and more specific inflammatory cell populations by the use of complementary markers, are certainly required. A potential candidate might be the invariant NKT that are suggested as a relevant $\mathrm{CD}^{+}$cell population in an animal model of NAFLD and fibrosis (53).

In conclusion, here we demonstrated that CD45, CD3 and CD163 intrahepatic expression correlated with the severity of 
disease in children with NAFLD. These results suggest that the assessment of the pattern of liver-resident and infiltration inflammatory cells may contribute not only to the diagnosis of NASH or to prediction of its potential progression to fibrosis, but also to identify novel pathways involved in the progression of the disease amenable to therapeutic intervention (54).

\section{Acknowledgements}

This study was entirely supported by Bambino Gesù Children's Hospital and Research Institute, Rome, Italy.

\section{References}

1. Alisi A, Manco M, Vania A and Nobili V: Pediatric nonalcoholic fatty liver disease in 2009. J Pediatr 155: 469-474, 2009.

2. Feldstein AE, Charatcharoenwitthaya P, Treeprasertsuk S, Benson JT, Enders FB and Angulo P: The natural history of nonalcoholic fatty liver disease in children: a follow-up study for up to 20 years. Gut 58: 1538-1544, 2009.

3. Papandreou D, Rousso I and Mavromichalis I: Update on nonalcoholic fatty liver disease in children. Clin Nutr 26: 409-415, 2007.

4. Dunn W and Schwimmer JB: The obesity epidemic and nonalcoholic fatty liver disease in children. Curr Gastroenterol Rep 10: 67-72, 2008

5. Brunt EM: Nonalcoholic steatohepatitis: definition and pathology. Semin Liver Dis 21: 3-16, 2001.

6. Patton HM, Lavine JE, Van Natta ML, Schwimmer JB, Kleiner D and Molleston J: Clinical correlates of histopathology in pediatric nonalcoholic steatohepatitis. Gastroenterology 135: 1961-1971, 2008.

7. Kim $\mathrm{CH}$ and Younossi ZM: Nonalcoholic fatty liver disease: a manifestation of the metabolic syndrome. Cleve Clin J Med 75: 721-728, 2008.

8. Pardee PE,Lavine JE and Schwimmer JB: Diagnosis and treatment of pediatric nonalcoholic steatohepatitis and the implications for bariatric surgery. Semin Pediatr Surg 18: 144-151, 2009.

9. Nobili V, Alisi A and Raponi M: Pediatric non-alcoholic fatty liver disease: preventive and therapeutic value of lifestyle intervention. World J Gastroenterol 15: 6017-6022, 2009.

10. de Alwis NM and Day CP: Non-alcoholic fatty liver disease: the mist gradually clears. J Hepatol 48 (Suppl 1): S104-S112, 2008.

11. Alisi A, Locatelli M and Nobili V: Nonalcoholic fatty liver disease in children. Curr Opin Clin Nutr Metab Care 13: 397-402, 2010.

12. Feldstein AE: Novel insights into the pathophysiology of nonalcoholic fatty liver disease. Semin Liver Dis 30: 391-401, 2010.

13. Tilg $\mathrm{H}$ and Moschen AR: Insulin resistance, inflammation, and non-alcoholic fatty liver disease. Trends Endocrinol Metab 19: 371-379, 2008.

14. Baffy G: Kupffer cells in non-alcoholic fatty liver disease: the emerging view. J Hepatol 51: 212-223, 2009.

15. Park JW, Jeong G, Kim SJ, Kim MK and Park SM: Predictors reflecting the pathological severity of non-alcoholic fatty liver disease: comprehensive study of clinical and immunohistochemical findings in younger Asian patients. J Gastroenterol Hepatol 22: 491-497, 2007.

16. Fotiadu A, Gagalis A, Akriviadis E, Kotoula V, Sinakos E, Karkavelas G and Hytiroglou P: Clinicopathological correlations in a series of adult patients with non-alcoholic fatty liver disease. Pathol Int 60: 87-92, 2010.

17. Rivera CA, Adegboyega P, van Rooijen N, Tagalicud A, Allman M and Wallace M: Toll-like receptor-4 signaling and Kupffer cells play pivotal roles in the pathogenesis of non-alcoholic steatohepatitis. J Hepatol 47: 571-579, 2007.

18. Bilzer M, Roggel F and Gerbes AL: Role of Kupffer cells in host defense and liver disease. Liver Int 26: 1175-1186, 2006.

19. Kolios G, Valatas V and Kouroumalis E: Role of Kupffer cells in the pathogenesis of liver disease. World J Gastroenterol 12 7413-7420, 2006

20. Zhan YT and An W: Roles of liver innate immune cells in nonalcoholic fatty liver disease. World J Gastroenterol 16: 4652-4660, 2010 .

21. Kremer M and Hines IN: Natural killer T cells and non-alcoholic fatty liver disease: fat chews on the immune system. World J Gastroenterol 14: 487-488, 2008.
22. Alisi A, Panera N and Nobili V: The link between hepatosteatosis and cells of the immune system. Hepatology 51: 1472-1473, 2010.

23. Alisi A, Panera N and Nobili V: Toll-like receptor 4: a starting point for proinflammatory signals in fatty liver disease. Hepatology 51: 714-715, 2010.

24. Spruss A, Kanuri G, Wagnerberger S, Haub S, Bischoff SC and Bergheim I: Toll-like receptor 4 is involved in the development of fructose induced hepatic steatosis in mice. Hepatology 50: 1094-1104, 2009

25. Kremer M, Thomas E, Milton RJ, et al: Kupffer cell and interleukin-12-dependent loss of natural killer T cells in hepatosteatosis. Hepatology 51: 130-141, 2010.

26. Stienstra R, Saudale F, Duval C, et al: Kupffer cells promote hepatic steatosis via interleukin-1beta-dependent suppression of peroxisome proliferator-activated receptor alpha activity. Hepatology 51: 511-522, 2010.

27. Torlakovic EE, Naresh K, Kremer M, van der Walt J, Hyjek E and Porwit A: Call for a European programme in external quality assurance for bone marrow immunohistochemistry; report of a European Bone Marrow Working Group pilot study. J Clin Pathol 62: 547-551, 2009.

28. Fabriek BO, Dijkstra CD and van den Berg TK: The macrophage scavenger receptor CD163. Immunobiology 210: 153-160, 2005.

29. Nguyen TT, Schwartz EJ, West RB, Warnke RA, Arber DA and Natkunam Y: Expression of CD163 (hemoglobin scavenger receptor) in normal tissues, lymphomas, carcinomas, and sarcomas is largely restricted to the monocyte/macrophage lineage. Am J Surg Pathol 29: 617-624, 2005.

30. Kuczmarski RJ, Ogden CL, Grummer-Strawn LM, et al: CDC growth charts: United States. Adv Data 314: 1-27, 2000.

31. Matsuda M and DeFronzo RA: Insulin sensitivity indices obtained from oral glucose tolerance testing: comparison with the euglycemic insulin clamp. Diabetes Care 22: 1462-1470, 1999.

32. Matthews DR, Hosker JP, Rudenski AS, Naylor BA, Treacher DF and Turner RC: Homeostasis model assessment: insulin resistance and beta-cell function from fasting plasma glucose and insulin concentrations in man. Diabetologia 28: 412-419, 1985.

33. Kleiner DE, Brunt EM, Van Natta M, et al: Nonalcoholic Steatohepatitis Clinical Research Network. Design and validation of a histological scoring system for nonalcoholic fatty liver disease. Hepatology 41: 1313-1321, 2005.

34. Alisi A, Bedogni G, De Vito R, Comparcola D, Manco M and Nobili V: Relationship between portal chronic inflammation and disease severity in paediatric non-alcoholic fatty liver disease. Dig Liver Dis 43: 143-146, 2011.

35. Valenti L, Fracanzani AL and Fargion S: The immunopathogenesis of alcoholic and nonalcoholic steatohepatitis: two triggers for one disease? Semin Immunopathol 31: 359-369, 2009.

36. Li Z, Soloski MJ and Diehl AM: Dietary factors alter hepatic innate immune system in mice with nonalcoholic fatty liver disease. Hepatology 42: 880-885, 2005.

37. de Luca C and Olefsky JM: Inflammation and insulin resistance. FEBS Lett 582: 97-105, 2008

38. Ma X, Hua J, Mohamood AR, Hamad AR, Ravi R and Li Z: A high fat diet and regulatory $\mathrm{T}$ cells influence susceptibility to endotoxin-induced liver injury. Hepatology 46: 1519-1529, 2007.

39. Shoelson SE, Herrero L and Naaz A: Obesity, inflammation, and insulin resistance. Gastroenterology 132: 2169-2180, 2007.

40. Tajiri K, Shimizu Y, Tsuneyama K and Sugiyama T: Role of liver infiltrating $\mathrm{CD}^{+} \mathrm{CD}^{+} 6^{+}$natural killer T cells in the pathogenesis of nonalcoholic fatty liver disease. Eur J Gastroenterol Hepatol 21: 673-680, 2009.

41. Notas G, Kisseleva T and Brenner D: NK and NKT cells in liver injury and fibrosis. Clin Immunol 130: 16-26, 2009.

42. Karlmark KR, Weiskirchen R, Zimmermann HW, et al: Hepatic recruitment of the inflammatory $\mathrm{Gr}^{+}$monocyte subset upon liver injury promotes hepatic fibrosis. Hepatology 50: 261-274, 2009.

43. Alisi A, Da Sacco L, Bruscalupi G, et al: Mirnome analysis reveals novel molecular determinants in the pathogenesis of dietinduced nonalcoholic fatty liver disease. Lab Invest 91: 283-293, 2011.

44. Holland-Fischer P, Grønbæk H, Sandahl TD, Moestrup SK, Riggio O, Ridola L, Aagaard NK, et al: Kupffer cells are activated in cirrhotic portal hypertension and not normalised by TIPS. Gut 60: 1389-1393, 2011.

45. Dolganiuc A, Norkina O, Kodys K, Catalano D, Bakis G, Marshall C, Mandrekar P, et al: Viral and host factors induce macrophage activation and loss of toll-like receptor tolerance inchronic HCV infection. Gastroenterology 133: 1627-1636, 2007. 
46. Hiraoka A, Horiike N, Akbar SM, Michitaka K, Matsuyama T and Onji M: Expression of CD163 in the liver of patients with viral hepatitis. Pathol Res Pract 201: 379-384, 2005.

47. Bauer S, Weiss TS, Wiest R, Schacherer D, Hellerbrand C, Farkas S, Scherer MN, et al: Soluble CD163 is not increased in visceral fat and steatotic liver and is even suppressed by free fatty acids in vitro. Exp Mol Pathol 91: 733-739, 2011.

48. Weaver LK, Hintz-Goldstein KA, Pioli PA, Wardwell K, Qureshi N, Vogel SN and Guyre PM: Pivotal advance: activation of cell surface Toll-like receptors causes shedding of the hemoglobin scavenger receptor CD163. J Leukoc Biol 80: 26-35, 2006.

49. Guebre-Xabier M, Yang S, Lin HZ, Schwenk R, Krzych U and Diehl AM: Altered hepatic lymphocyte subpopulations in obesity-related murine fatty livers: potential mechanism for sensitization to liver damage. Hepatology 31: 633-640, 2000.

50. Kremer M, Hines IN, Milton RJ and Wheeler MD: Favored T helper 1 response in a mouse model of hepatosteatosis is associated with enhanced T cell-mediated hepatitis. Hepatology 44: 216-227, 2006
51. Xu CF, Yu CH, Li YM, Xu L, Du J and Shen Z: Association of the frequency of peripheral natural killer $\mathrm{T}$ cells with nonalcoholic fatty liver disease. World J Gastroenterol 13: 4504-4508, 2007.

52. Eberl G and MacDonald HR: Rapid death and regeneration of NKT cells in anti-CD3epsilon- or IL-12-treated mice: a major role for bone marrow in NKT cell homeostasis. Immunity 9: 345-353, 1998.

53. Miyagi T, Takehara T, Uemura A, et al: Absence of invariant natural killer $\mathrm{T}$ cells deteriorates liver inflammation and fibrosis in mice fed high-fat diet. J Gastroenterol 45: 1247-1254, 2010.

54. Iacono A, Raso GM, Canani RB, Calignano A and Meli R: Probiotics as an emerging therapeutic strategy to treat NAFLD: focus on molecular and biochemical mechanisms. J Nutr Biochem 22: 699-711, 2011. 